\section{Uso do cartão da criança em Feira de Santana, Bahia}

\section{Children's care cards use in Feira de Santana, Bahia}

Graciete Oliveira Vieira 1

Tatiana de Oliveira Vieira 2

Maria Conceição Oliveira Costa 3

Pedro Vieira Santana Netto 4

Vilma Alves Cabral 5

${ }^{1}$ Departamento de Saúde. Núcleo de Estudos e Pesquisas na Infância e Adolescência da Universidade Estadual de Feira de Santana. Campus Universitário, Km. 03, BR 116. CEP: 44.031-460. Feira de Santana, BA, Brasil. http://www.uefs.br

2,4 Faculdade de Medicina de São José do Rio Preto, SP, Brasil. 3 Departamento de Saúde. Programa de Pós Graduação em Saúde Coletiva. Núcleo de Estudos e Pesquisas na Infância e

Adolescência Universidade Estadual de Feira de Santana, BA, Brasil.

5 Fundo das Nações Unidas para a Infância. Salvador, BA, Brasil.

\section{Resumo}

Objetivos: verificar o indice de preenchimento do "Cartão da Criança (CC)" com ênfase no acompanhamento do crescimento e desenvolvimento em menores de um ano em Feira de Santana, Bahia, 2001.

Métodos: estudo transversal. Foram aplicados 2319 questionários às mães das crianças menores de um ano, nas 44 Unidades de Saúde selecionadas por estratificação simples e utilizadas medidas de significância estatística, através do qui-quadrado com $p-5,0 \%$. Foi calculada razão de prevalência e intervalo de confiança $95 \%$.

Resultados: das mães entrevistadas 95,5\% portavam CC. O indice Apgar constava em 28,4\% dos CC. Quanto às curvas de crescimento e de desenvolvi mento $39,6 \%$ e 77,9\% respectivamente não foram preenchidas. Filhos de mães que não trabalham fora do lar elou com renda mensal menor que dois salários minimos apresentaram chance significantemente maior de ter o peso anotado no CC. Primiparidade e escolaridade materna maior que o ensino básico fundamental esteve associado a maior probabilidade de anotação da curva de desenvolvimento. Residir na área de atuação dos agentes comunitários de saúde e idade inferior a seis meses mostraram-se fatores de proteção para preenchimento da curva de desenvolvi mento.

Conclusões: na população estudada, o CC não estava sendo utilizado plenamente, principalmente nos dados de desenvolvimento e índice de Apgar.

Palavras-chave Crescimento, Desenvolvimento infantil, Bem-estar da criança, Promoção da saúde. 


\section{Introdução}

As crianças, por serem um complexo biológico em formação, estão mais vulneráveis aos condicionantes ambientais, os quais podem interferir nos índices de morbidade e de mortalidade, assim como comprometer o processo de crescimento e desenvolvimento e a expressão do potencial genético.1-5

A monitorização do crescimento foi recomendada desde a década de 70, na Conferência de AlmaAta, realizada no Cazaquistão, em 1978. No Brasil, embora o relatório da V Conferência Nacional de Saúde, realizada nessa mesma década, tenha incorporado a vigilância do crescimento, o tema somente ganhou relevância em 1984, com a criação do "Programa de Assistência Integral à Saúde da Criança (PAISC)" pelo Ministério da Saúde. As ações básicas de saúde formuladas pelo PAISC visam assegurar integralidade na assistência prestada pelos serviços de saúde, deslocando o enfoque da atenção voltada às patologias para a priorização das ações preventivas, cujo eixo básico é garantir o adequado crescimento e desenvolvimento.6-8

As cinco ações básicas propostas pelo PAISC são: a) o incentivo ao aleitamento materno e orientação adequada para o desmame; b) assistência e controle das infecções respiratórias agudas; c) imunização para controle das doenças preveníveis; d) controle das doenças diarréicas e e) acompanhamento do crescimento e desenvolvimento (CD) infantil, $, 9,10$ tendo como meio de visualização o "Cartão da Criança (CC)".10,11

O CC utiliza o aumento mensal do peso como principal indicador do crescimento normal e sadio da criança, ou seja, uma curva de crescimento com pontos de cortes e padrões de referências que permite aos profissionais de saúde e aos pais acompanharem o CD das crianças menores de cinco anos. Os cartões de meninos e meninas possuem apresentações diferentes, uma vez que os padrões de crescimento são desiguais. ${ }^{12}$

Outro aspecto importante é que o CC é um instrumento de caráter educativo e se constitui numa maneira econômica, não-invasiva, de fácil interpretação e compreensão por parte da comunidade. Os pais ou responsáveis, em poder do cartão, têm a oportunidade de observar e acompanhar o CD de seus filhos, elemento indispensável para identificação precoce de problemas de saúde dinâmicos, como a desnutrição e retardo do crescimento, com possibilidade de reversão do processo, em tempo de garantir um crescimento adequado à criança, uma vez que quadros crônicos, quando instalados, tendem a promover repercussões irreversíveis. $8,13,14$ Vale ressaltar que a antropometria é a técnica mais universalmente aplicada para avaliar o tamanho e representa um indicador de crescimento e de risco nutricional. Pela sua capacidade de monitorar saúde e crescimento, tem lugar de destaque na Saúde Publica, especialmente no caso de países em desenvolvimento. 15 Entretanto, a utilização de medidas antropométricas pode diferir significativamente, de acordo com o objeto de estudo, tanto do ponto de vista individual quanto coletivo15,16 e deve ser complementada por outras informações de saúde, nutrição, culturais, socioeconômicas e demográficas. 1,15

O "Cartão da Criança", além de monitorar o ganho ponderal, permite também o registro de identificação da criança, tipo de parto, índice de Apgar, local e data de nascimento, calendário para anotação das vacinas, informações básicas de desenvolvimento e dos direitos constitucionais das crianças. $\mathrm{O}$ "Cartão" é válido em todo o território nacional como comprovante de vacinação e o seu fornecimento é gratuito e deve ocorrer no momento do nascimento, com a posse assegurada aos pais.

Com a promulgação da Constituição de 1988 houve um implemento das ações preventivas, o que determinou a decisão de implantação do CC em todo território nacional. Em 1989, a Secretaria de Saúde da Bahia implantou as ações básicas de saúde e normalizou a utilização do CC para acompanhamento de saúde de menores de cinco anos, em todo estado.

Visando estender as ações básicas de saúde aos núcleos familiares, em 1991, o Ministério da Saúde oficializou o Programa de Agentes Comunitários de Saúde (PACS), com uma agenda prioritariamente direcionada ao grupo materno-infantil. Em Feira de Santana, o PACS foi implantado em 1992.17

No ano de 2005, o "Cartão da Criança" foi revisado pelo Ministério da Saúde, resultando na "Caderneta de saúde da criança", com ampliação da faixa de acompanhamento para dez anos de idade. Nesse novo instrumento, além das informações contidas no $\mathrm{CC}$, foram incluídos dados sobre gravidez, parto e puerpério; orientações relevantes sobre a alimentação saudável, gráficos de perímetro cefálico, espaço para anotações de estatura, informações quanto à prevenção de acidentes, à saúde auditiva, visual e bucal, profilaxia de ferro e vitamina A e espaço para anotações de intercorrências clínicas. ${ }^{18,19}$

Considerando a importância desses instrumentos para a avaliação e acompanhamento de saúde das crianças, o presente estudo teve como objetivo analisar o índice de preenchimento do "Cartão da Criança", assim como possíveis associações com variáveis 
maternas e das crianças menores de um ano, residentes no município de Feira de Santana, Bahia, em 2001.

\section{Métodos}

Trata-se de um estudo observacional de corte transversal, aprovado pelo Comitê de Ética e Pesquisa da Universidade Federal da Bahia, realizado na cidade de Feira de Santana, Bahia, situada a $108 \mathrm{Km}$ da capital do estado - Salvador - com uma população em torno de 480.949 habitantes. 17

Foram selecionadas crianças menores de um ano que haviam completado essa idade até o dia 25 de agosto de 2001 (Dia Nacional de Vacinação), procedentes de Feira de Santana e que compareceram às unidades de vacinação sorteadas, acompanhadas de suas respectivas mães, as quais aceitaram responder ao questionário. A unidade de análise correspondeu às mães e crianças menores de um ano, selecionadas para a amostra.

O plano amostral foi probabilístico e estratificado. Para assegurar a cobertura vacinal, baseada na demanda de crianças atendidas em cada unidade de saúde, na primeira etapa de vacinação a Secretária de Saúde de Feira de Santana dividiu a cidade em quatro áreas geográficas (comandos), representadas por bairros que correspondiam a subpopulações mutuamente excludentes; desta forma, uma amostra casual simples foi retirada de cada estrato, levando-se em consideração o número de crianças vacinadas por área, na primeira etapa de vacinação. A vacinação ocorreu em 62 unidades (postos de saúde e escolas) e foram sorteadas 44 unidades para compor a amostra. Do primeiro e segundo estratos (comandos) foram sorteadas respectivamente 12 dentre as 17 unidades existentes e do terceiro e quarto comandos foram sorteadas respectivamente 10 entre as 14 unidades. Responderam ao questionário 2319 mães, correspondendo a $24,1 \%$ da população (9615) de crianças menores de um ano, residentes no município de Feira de Santana, em 2001.17 Apenas quatro mães se recusaram a participar da pesquisa.

Foram aplicados questionários individuais, prétestados durante o plano piloto. As questões elaboradas foram fechadas, com três alternativas excludentes: sim, não, não sei/não lembro e aplicadas diretamente às mães, não sendo coletadas informações de terceiros, evitando assim a interferência do efeito proxy. Foram também coletadas informações diretamente do CC para informações como peso, perímetro cefálico, comprimento e índice de Apgar ao nascimento.
O questionário foi aplicado por 104 universitários da área de saúde, previamente treinados. O treinamento foi realizado em duas fases. A primeira fase consistiu no treinamento de oito internos e residentes de medicina, como supervisores e na segunda fase foram treinados 96 estudantes de odontologia e enfermagem, divididos em cinco grupos. O treinamento consistiu em apresentação da pesquisa pelo coordenador, apresentação do questionário, realização de leitura do questionário e do manual do entrevistador e aplicação do questionário, individualmente, ao colega e vice-versa. Todos os inquiridores realizaram pelo menos quatro leituras do questionário, antes de ir para o trabalho de campo. Só foi permitido realizar a pesquisa os alunos que participaram de todas as etapas do treinamento.

As variáveis estudadas, em relação às características maternas, foram: idade, paridade, escolaridade, renda mensal familiar, trabalho fora do lar e local de residência. No que se refere aos dados das crianças, foram coletadas informações, conforme o preenchimento do $\mathrm{CC}$, quanto à data de nascimento para cálculo da idade, sexo, idade gestacional, tipo de parto, índice de Apgar do $5^{\circ}$ minuto de vida, peso, comprimento e perímetro cefálico por ocasião do nascimento. Observadas também anotações no CC quanto ao nome do hospital em que a criança nasceu e o monitoramento do CD.

Quanto às anotações presentes na identificação do $\mathrm{CC}$, foram consideradas como incompletas a ausência de um dos itens. Para a curva de crescimento, foi considerada completa mediante a anotação de pesos pelo menos a cada três meses, com um mínimo de quatro anotações no primeiro ano de vida; enquanto que a curva de desenvolvimento foi considerada como completa quando o cartão possuía todas as anotações correspondentes à idade da criança entrevistada.

$\mathrm{Na}$ análise para avaliação das associações entre as variáveis, calculou-se a razão de prevalência (RP) e, para inferência estatística, efetuou-se o teste do quiquadrado, com cálculo de valor de $p$ e do Intervalo de Confiança 95\% (IC95\%), sendo considerados como estatisticamente significantes valores bicaudais de $p$ iguais ou menores do que $5,0 \%$. O programa estatístico utilizado foi o Statistical Package for Social Sciences (SPSS), na versão 10.0.

\section{Resultados}

O total de crianças analisadas não perfaz 2319 para todas as questões, porque foram consideradas como válidas apenas as repostas positivas ou negativas, 
sendo computadas como perdidas/inválidas as questões respondidas como "não sei/não lembro" e as incompletas.

Das 2319 mães de crianças menores de um ano entrevistadas, $95,5 \%$ (2215) portavam o CC. No que se refere às anotações do nome da criança e dos pais, não constavam simultaneamente em $36,5 \%$ dos cartões, sendo considerados incompletos, embora o nome da criança estivesse anotado em $99,8 \%$. O nome do hospital de nascimento constava em 76,6\%; o tipo de parto em $93,3 \%$ e o índice de Apgar do $5^{\circ}$ minuto após o nascimento em apenas $28,4 \%$ dos cartões. As variáveis, peso, perímetro cefálico e comprimento por ocasião do nascimento, estavam presentes em $97,2 \%, 88,9 \%$ e $91,8 \%$ dos cartões, respectivamente.

O número de crianças na faixa etária de 30 dias variou de $6,9 \%(161)$ a $10,3 \%,(240)$ e dentre as 2319 crianças estudadas $1224 \quad(52,8 \%)$ eram menores ou iguais a seis meses. As características maternas e das crianças pesquisadas estão descritas na Tabela 1 .

Tabela 1

Distribuição das variáveis referentes às características maternas e das crianças.

\begin{tabular}{|c|c|c|}
\hline Variável & $\mathbf{N}$ & $\%$ \\
\hline Idade materna & 2314 & 100,0 \\
\hline$<20$ anos & 513 & 22,2 \\
\hline$\geq 20$ anos & 1801 & 77,8 \\
\hline Paridade & 2318 & 100,0 \\
\hline Multípara & 1246 & 53,8 \\
\hline Primípara & 1072 & 46,2 \\
\hline Trabalho fora do lar & 2315 & 100,0 \\
\hline Não & 1776 & 76,7 \\
\hline Sim & 539 & 23,3 \\
\hline Escolaridade & 2308 & 100,0 \\
\hline Básico fundamental & 638 & 27,7 \\
\hline >Básico fundamental & 1670 & 72,3 \\
\hline Renda familiar & 1640 & 100,0 \\
\hline -2 salários mínimos & 1038 & 63,3 \\
\hline >2 salários mínimos & 602 & 36,7 \\
\hline Peso de nascimento & 2258 & 100,0 \\
\hline$<2500$ gramas & 165 & 7,3 \\
\hline-2500 gramas & 2093 & 92,7 \\
\hline Prematuridade & 2314 & 100,0 \\
\hline Sim & 172 & 7,4 \\
\hline Não & 2142 & 92,6 \\
\hline
\end{tabular}


Com relação às 2200 respostas válidas, quanto ao preenchimento da curva de crescimento segundo as categorias completa, incompleta e inexistente, as prevalências foram respectivamente $41,1 \%$ (905), $29,3 \%$ (644) e 39,6\% (651). Em relação à curva de desenvolvimento, das 2191 crianças cujos cartões foram avaliados, $77,9 \%$ (1707) não foram preenchidos, $14,3 \%$ (314) estavam incompletos e $7,8 \%$ (170) completos.

Em relação à curva de crescimento foram observadas associações estatisticamente significantes, com maior probabilidade para o seu preenchimento quando as crianças eram filhos de família com renda mensal menor que dois salários mínimos ou quando as mães não se ausentavam do lar para trabalhar. Observou-se associação, no sentido da proteção entre a idade da criança menor que seis meses e o preenchimento dessa curva. As demais associações estudadas não apresentaram níveis de significância estatística (Tabela 2).

Com respeito ao preenchimento da curva de desenvolvimento, verificou-se associação positiva para o preenchimento desses dados nos casos de mães primíparas e com nível de instrução superior ao ensino básico fundamental (Tabela 3). Houve também uma associação, no sentido da proteção, entre morar em área de atuação do PACS, ter idade menor que seis meses e ter a curva de desenvolvimento anotada no CC (Tabela 3). As demais variáveis estudadas não apresentaram associações em níveis de significância estatística (Tabela 3).

Tabela 2

Razão de prevalência (RP) e intervalo de confiança de 95\% (IC95\%) da associação entre preenchimento da curva de crescimento nos cartões da criança e características maternas.

\begin{tabular}{|c|c|c|c|c|c|c|c|c|}
\hline \multirow{3}{*}{ Variável } & \multicolumn{8}{|c|}{ Cuna de crescimento } \\
\hline & \multicolumn{2}{|c|}{ Sim } & \multicolumn{2}{|c|}{ Não } & \multicolumn{2}{|c|}{ Total } & \multirow{2}{*}{ RP $\quad(I C 95 \%)$} & \multirow{2}{*}{$p$} \\
\hline & $\mathrm{n}$ & $\%$ & $\mathrm{n}$ & $\%$ & $\mathrm{n}$ & $\%$ & & \\
\hline \multicolumn{9}{|l|}{ Parto prematuro } \\
\hline Não & 1431 & 70,1 & 609 & 29,9 & 2040 & 93,0 & $0,96(0,87-1,06)$ & 0,40 \\
\hline Sim & 113 & 73,4 & 41 & 26,6 & 154 & 7,0 & & \\
\hline \multicolumn{9}{|l|}{ Paridade } \\
\hline Primípara & 725 & 71,5 & 289 & 28,5 & 1014 & 46,1 & $1,03(0,98-1,09)$ & 0,29 \\
\hline Multípara & 823 & 69,5 & 362 & 30,5 & 1185 & 53,9 & & \\
\hline \multicolumn{9}{|l|}{ Idade materna } \\
\hline$<20$ anos & 351 & 72,2 & 135 & 27,8 & 486 & 22,1 & $1,03(0,97-1,10)$ & 0,32 \\
\hline$\geq 20$ anos & 1198 & 69,9 & 516 & 30,1 & 1714 & 77,9 & & \\
\hline \multicolumn{9}{|l|}{ Escolaridade* } \\
\hline >Fundamental & 1118 & 70,8 & 462 & 29,2 & 1580 & 72,2 & $1,02(0,96-1,08)$ & 0,60 \\
\hline Fundamental & 424 & 69,6 & 185 & 30,4 & 609 & 27,8 & & \\
\hline \multicolumn{9}{|l|}{ Trabalho fora do lar } \\
\hline Não & 1207 & 71,8 & 474 & 28,2 & 1681 & 76,6 & $1,09(1,02-1,17)$ & 0,01 \\
\hline $\operatorname{Sim}$ & 340 & 66,0 & 175 & 34,0 & 515 & 23,4 & & \\
\hline \multicolumn{9}{|l|}{ Renda familiart } \\
\hline <2 salários mínimos & 713 & 73,1 & 263 & 26,9 & 976 & 63,0 & $1,01(1,00-1,14)$ & 0,05 \\
\hline 22 salários mínimos & 392 & 68,3 & 182 & 31,7 & 574 & 37,0 & & \\
\hline \multicolumn{9}{|l|}{ Mora na área do PACS $\ddagger$} \\
\hline $\operatorname{sim}$ & 1074 & 69,9 & 468 & 30,4 & 1542 & 70,4 & $0,96(0,91-1,02)$ & 0,19 \\
\hline Não & 470 & 72,4 & 179 & 27,6 & 649 & 29,6 & & \\
\hline \multicolumn{9}{|l|}{ Idade da criança } \\
\hline$<6$ meses & 665 & 58,6 & 470 & 41,4 & 1135 & 51,6 & $0,70(0,67-0,74)$ & 0,001 \\
\hline$\geq 6$ meses & 884 & 83,0 & 181 & 17,0 & 1065 & 48,4 & & \\
\hline
\end{tabular}

* Ensino básico fundamental; † Renda familiar em salários mínimos; ¥ Programa Agente Comunitário de Saúde. 
Razão de prevalência (RP) e intervalo de confiança de $95 \%$ (IC95\%) da associação entre preenchimento da curva de desenvolvimento nos cartões da criança e características maternas.

\begin{tabular}{|c|c|c|c|c|c|c|c|c|}
\hline \multirow{3}{*}{ Variável } & \multicolumn{8}{|c|}{ Curva de desenvolvimento } \\
\hline & \multicolumn{2}{|c|}{ Sim } & \multicolumn{2}{|c|}{ Não } & \multicolumn{2}{|c|}{ Total } & \multirow{2}{*}{ RP e IC95\% } & \multirow{2}{*}{$p$} \\
\hline & $\mathrm{n}$ & $\%$ & $\mathrm{n}$ & $\%$ & $\mathrm{n}$ & $\%$ & & \\
\hline \multicolumn{9}{|l|}{ Parto prematuro } \\
\hline Não & 456 & 22,4 & 1576 & 77,6 & 2032 & 93,0 & $1,27(0,90-1,81)$ & 0,17 \\
\hline Sim & 27 & 17,6 & 126 & 82,4 & 153 & 7,0 & & \\
\hline \multicolumn{9}{|l|}{ Paridade } \\
\hline Primípara & 243 & 24,1 & 766 & 75,9 & 1009 & 46,1 & $1,18(1,01-1,38)$ & 0,04 \\
\hline Multípara & 241 & 20,4 & 940 & 79,6 & 1181 & 53,9 & & \\
\hline \multicolumn{9}{|l|}{ Idade materna } \\
\hline$<20$ anos & 111 & 22,9 & 374 & 77,1 & 485 & 22,1 & $1,05(0,87-1,26)$ & 0,63 \\
\hline$\geq 20$ anos & 373 & 21,9 & 1333 & 78,1 & 1706 & 77,9 & & \\
\hline \multicolumn{9}{|l|}{ Escolaridade* } \\
\hline >Fundamental & 368 & 23,4 & 1206 & 76,6 & 1574 & 72,2 & $1,22(1,01-1,47)$ & 0,03 \\
\hline Fundamental & 116 & 19,1 & 490 & 80,9 & 606 & 27,8 & & \\
\hline \multicolumn{9}{|l|}{ Trabalho fora do lar } \\
\hline Não & 382 & 22,8 & 1291 & 77,2 & 1673 & 76,5 & $1,15(0,95-1,40)$ & 0,15 \\
\hline $\operatorname{Sim}$ & 102 & 19,8 & 412 & 80,2 & 514 & 23,5 & & \\
\hline \multicolumn{9}{|l|}{ Renda familiart } \\
\hline <2 salários mínimos & 225 & 23,1 & 750 & 76,9 & 975 & 63,1 & $0,97(0,81-1,17)$ & 0,77 \\
\hline 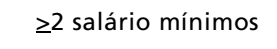 & 135 & 23,7 & 434 & 76,3 & 569 & 36,9 & & \\
\hline \multicolumn{9}{|l|}{ Mora na área do PACS } \\
\hline $\operatorname{Sim}$ & 304 & 19,8 & 1233 & 80,2 & 1537 & 70,4 & $0,71(0,61-0,84)$ & 0,001 \\
\hline Não & 179 & 27,8 & 466 & 72,2 & 645 & 29,6 & & \\
\hline \multicolumn{9}{|l|}{ Idade da criança } \\
\hline$<6$ meses & 204 & 18,0 & 930 & 82,0 & 1134 & 51,8 & $0,68(0,58-0,80)$ & 0,001 \\
\hline$\geq 6$ meses & 280 & 26,5 & 777 & 73,5 & 1057 & 48,2 & & \\
\hline
\end{tabular}

* Ensino básico fundamental; † Renda familiar em salários mínimos; ¥ Programa Agente Comunitário de Saúde.

\section{Discussão}

Diante do recente cenário em que figura a implantação da nova "Caderneta de Saúde da Criança" do Ministério da Saúde em 2005, documento esse que amplia as informações contidas no "Cartão da Criança" e requer maior participação dos pais e compromisso dos profissionais de saúde quanto ao preenchimento, ressalta-se a importância de avaliar a utilização do "Cartão da Criança" pelos pais e profissionais de saúde. Em Feira de Santana foi constatado que, apesar da quase totalidade das mães entrevistadas portar o CC, no "Dia Nacional de Vacinação", a maior parte dos cartões não estavam preenchidos de forma plena.
Foram constatadas ausências de anotações em relação ao nome da criança e dos pais, hospital em que a criança nasceu, tipo de parto, peso, perímetro cefálico e comprimento por ocasião do nascimento. A Pesquisa Nacional de Demografia e Saúde (PNDS), realizada no Brasil, em 1996, demonstrou resultados semelhantes, na qual a maioria das mães possuía o cartão e levava-o às consultas nos serviços de saúde, porém, em menos de $10 \%$ constava o peso da criança e uma proporção ainda menor possuía a curva de crescimento desenhada. ${ }^{6}$ Santos et al.,20 realizaram também uma pesquisa em "Dia Nacional de Vacinação", com 329 mães de crianças menores de um ano, na qual verificaram que $99,7 \%$ alegavam possuir o $\mathrm{CC}$, embora 
$6,7 \%$ não o portassem na ocasião e $30 \%$ das crianças que compareceram à consulta pediátrica, nos três meses que antecederam a pesquisa, não possuíam qualquer registro de peso no período. 20

$\mathrm{Na}$ presente pesquisa, outro aspecto relevante foi a ausência da anotação do índice Apgar na grande maioria dos cartões, fato que chama a atenção para a possibilidade de as crianças não estarem sendo assistidas adequadamente ao nascimento, com conseqüente inexistência dessa anotação. Esse índice está associado à vitalidade do recém-nascido, nos primeiros momentos de vida, em que condições adversas ocorridas ao nascimento e refletidas em baixos valores de Apgar podem ser identificadas para que medidas de intervenção possam ser tomadas precocemente, através de técnicas de estimulação, para prevenção de possíveis seqüelas.

A falta ou inadequação no preenchimento por parte da equipe de saúde dos CC é injustificável e demonstra um fraco vínculo dos profissionais com as ações básicas de saúde, medidas de comprovada eficácia, haja vista a contribuição dessas ações no perfil de mortalidade infantil ocorrido nas duas últimas décadas.4,5 Vale ressaltar que, para prover o sucesso de implantação da nova "Caderneta de saúde da criança", tendo como foco os profissionais de saúde, o Ministério da Saúde elaborou um Manual para sua utilização que, além de orientar o correto preenchimento dos dados, reforça a importância do profissional como educador e promotor da saúde e ressalta a "Caderneta" como um direito que não pode ser negado à criança. 19 Resta saber se esse instrumento terá impacto suficiente para modificação das atitudes e práticas dos profissionais de saúde. Para Santos et $a l ., 20$ as dificuldades para a implementação de novas ações de saúde concentram-se também na falta de motivação, na dificuldade de mudança de comportamento e de atitudes por parte da equipe de saúde.

$\mathrm{Na}$ presente pesquisa, um achado importante foi a associação positiva, estatisticamente significante, entre renda familiar mensal inferior a dois salários mínimos e a realização da curva de crescimento, fato que pode estar relacionado à priorização de ação do PACS nas áreas em que residem comunidades menos privilegiadas do ponto de vista socioeconômico e com menor acesso às unidades de saúde. A anotação do peso da criança no $\mathrm{CC}$ é realizada pelos próprios agentes de saúde, após a sua aferição.

Em relação às anotações sobre o desenvolvimento neuropsicomotor, notou-se ausência de anotações na grande maioria dos CC, apesar de ter sido observada uma associação no sentido da proteção entre residir na área de atuação do PACS e o preenchimento dessa curva. Outra associação no sentido da proteção para o preenchimento, não só da curva de desenvolvimento como também a de crescimento, foi a idade da criança menor que seis meses. Esse resultado já era esperado, uma vez que existe uma tendência natural de maior atenção e cuidado à saúde das crianças de menor idade, não só por parte dos pais, como também pelos profissionais de saúde. Neste estudo, foi demonstrado também que o nível de instrução da mãe influencia no preenchimento da curva de desenvolvimento, em que mães com escolaridade superior ao ensino básico fundamental apresentaram uma associação positiva estatisticamente significante quanto à sua elaboração. Em verdade, é relevante a participação dos pais nesse processo, uma vez que são eles que constatam as etapas de desenvolvimento atingidas pela criança. Entretanto, os profissionais de saúde são os responsáveis pelo acompanhamento do desenvolvimento da criança e da sensibilização dos pais quanto ao preenchimento do cartão.

Ainda sobre a curva de desenvolvimento, os achados desse estudo mostraram que mães primíparas tiveram maior atenção quanto ao preenchimento. É provável que mães inexperientes, na maior parte primíparas, busquem informações junto à família, à comunidade e aos profissionais de saúde. Segundo Carvalho Filho,13 a percepção materna do CD dos filhos por indicadores próprios é uma realidade controversa, visto que a mãe da criança, na maioria das vezes, usa o CC apenas como cartão de vacinação e só demonstra interesse no gráfico perante problema de saúde do filho. Entretanto, é importante lembrar que é possível que as mães não reconheçam a necessidade de sua participação na avaliação do CD de seus filhos, por conta de que, habitualmente, as ações de saúde sempre foram delegadas aos profissionais, não sendo permitida ou estimulada a interferência ativa das mães nesse processo.

\section{Conclusões e considerações}

Baseado nos resultados dessa pesquisa conclui-se que quase a totalidade das mães portava o "Cartão" no "Dia Nacional da Vacinação" e que a maioria destes tinha a curva de crescimento preenchida. Entretanto, esse instrumento não estava sendo utilizado de maneira adequada em relação ao acompanhamento das etapas do desenvolvimento e à anotação do índice de Apgar. As variáveis significativamente associadas ao preenchimento da curva de crescimento foram: mães que não trabalhavam fora do lar ou famílias com menor renda. Em relação à curva de desenvolvimento, foi notada maior probabilidade para a sua realização quando as mães eram 
primíparas ou com maior escolaridade ou residentes na área de atuação do PACS. A idade da criança menor que seis meses figurou como fator de proteção tanto para o preenchimento da curva de crescimento, quanto à de desenvolvimento.

A concepção de que a atenção integral à saúde da criança, por parte dos profissionais de saúde e dirigentes, deve ir além dos gráficos não pode ser perdida. Entende-se que a utilização plena do "Cartão da Criança", para as aquelas que nasceram até o ano de 2004, assim como a "Caderneta de saúde da criança" (adotada a partir de 2005), são instrumentos essenciais para promover a vigilância à saúde integral da criança e que necessitam da participação e compromisso dos profissionais de saúde para a sua implementação. Fica assim evidente a necessidade de uma reflexão por parte dos dirigentes e profissionais de saúde acerca das rotinas dos serviços de saúde, otimizando medidas de intervenção que possibilitem o cumprimento das metas de implantação do CC no município. A utilização desses instrumentos conforme o preconizado constitui um importante indicador da qualidade da atenção oferecida pelos serviços de saúde às crianças.

\section{Referências}

1. Barros FC, Victora CG. Epidemiologia da saúde infantil. São Paulo: HUCITEC; 1998.

2. Black RE, Morris SS, Bryce J. Where and why are 10 million children dying every year? The Lancet 2003; 9376: 2226-34.

3. Eisenstein E. Antropometria e pediatria. J Pediatr (Rio J) 1994; 70 (4):193-4.

4. UNICEF (Fundo das Nações Unidas para a Infância). A infância brasileira nos anos 90. Brasília (DF); 1998.

5. Monteiro CA, Nazário CL. Declínio da mortalidade infantil e equidade social: o ca so da cidade de S ão Paulo entre 1973 e 1993. In: Monteiro CA, editor. Velhos e novos males da saúde no Brasil: a evolução do país e de suas doenças. São Paulo: Hucitec; 1995. p.173-85.

6. Ministério da Saúde. Crescimento e desenvolvimento [on line]. Disponível em URL: http://www.saude.gov.br/ programas/scriança/criança/crescimento.htm [2002 jul 6]

7. Ministério da Saúde, CONASEMS (Conselho Nacional dos Secretários Municipais de Saúde). Plano de ação para a implantação da declaração mundial sobre a sobrevivência, a proteção e o desenvolvimento da criança nos anos 90. In: Ministério da Saúde. Metas - Projeto Município Amigo da Criança. Brasília (DF); 1990.

8. Barreto ML, Carma EH. Situação da saúde da população brasileira: tendências históricas,

9. Ministério da Saúde. Centro de Documentação. Assistência integral à saúde da criança: ações básicas. Brasília (DF); 1984. (Textos Básicos de Saúde. Série B, 7).

10. Ministério da Saúde. Situação da saúde da criança no Brasil [on line]. Disponível em URL: http://www.saude.gov.br/ programas/scriança/hpcriança.htm [2002 jul 6].

11. Marcondes E. Crescimento normal: tabelas e gráficos. In: Marcondes E. Crescimento normal e deficiente. São Paulo:

Recebido em 26 de junho de 2004

Versão final apresentada em 4 de abril de 2005

Aprovado em 25 de abril de 2005
Savier; 1989. p. 42-69.(Monografias médicas. Serie Pediatria, v.1).

12. Costa MCO. Crescimento e desenvolvimento na infância e na adolescência. In: Costa S, editor. Semiologia e atenção primária ás crianças e adolescentes. Porto Alegre: Revinter; 2005. p. 17-39.

13. Carvalho Filho GWB. Crescendo e aparecendo: o crescimento infantil na percepção materna [dissertação mestrado]. Rio de Janeiro: Instituto Fernandes Figueira da Fundação Oswaldo Cruz; 1991.

14. Marcondes E. Monitorização do crescimento. In: Marcondes E. Crescimento normal e deficiente. São Paulo: Sarvier; 1989. p. 5-32. (Monografias médicas. Serie Pediatria, 1).

15. Accioly E, Meireles CL, Valle J, Franceschini SCC, Taddei JAAC, Sigulem DM. Antropometria na infância. Ars Cvrandi 1999; 5: 44- 52.

16. Marques RFSV, Lopes FA, Braga JA. O crescimento de crianças alimentadas com leite materno exclusivo nos primeiros seis meses de vida. J Pediatr (Rio J) 2004; 80: 99-105.

17. Feira de Santa. Secretaria de Saúde. Núcleo de Informações e Pesquisas Econômicas e Sociais. Disponível em URL: http://www.nipes.feiradesantana.ba.gov.br/f_dados.htm [2004 jul 13].

18. Ministério da Saúde. Departamento de Informação e Informática do SUS. Disponível em URL: http://tabnet. datasus.gov.br/cgi/tabcgi.e xe?ibge/cnv/popba. def [2005 fev 22].

19. Ministério da Saúde. Secretária de Atenção à Saúde. Departamento de Ações Programáticas Estratégicas. Manual para utilização da caderneta de saúde da criança. Brasília (DF); 2005.

20. Santos RS, Cunha AJLA, Gambá CM, Machado FG, Leal Filho JMM, Moreira NLM. Avaliação da assistência à saúde da mulher e da criança em localidade urbana da região Sudeste do Brasil. Rev Saúde Pública 2000; 34: 266- 71. 